\title{
Comparative Evaluation of Antimicrobial Activity of Quercetin against Endodontic Pathogens in Primary Molars
}

\author{
Shagun Sinha ${ }^{1}$, Roopa Korishettar ${ }^{2}$, Poornima Parameswarappa ${ }^{3}$, Neena I Eregowda ${ }^{4}$, Mallikarjun Kenchappa ${ }^{5}$, \\ Shilpa Sasalawad ${ }^{6}$
}

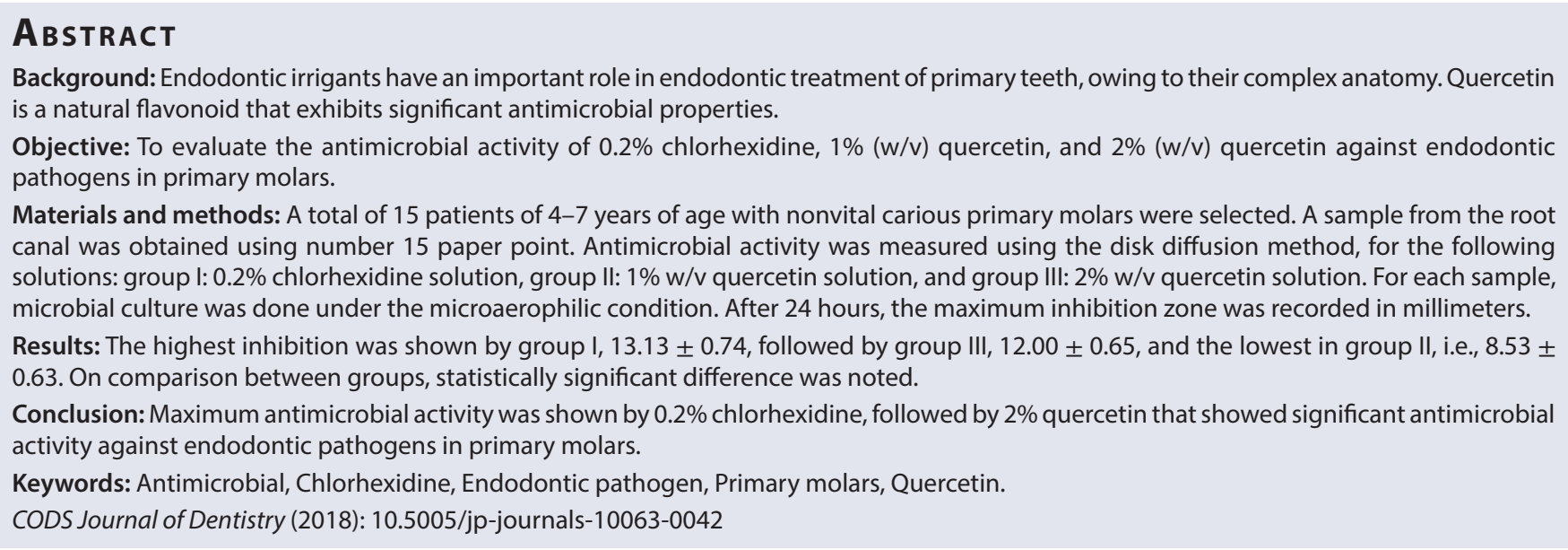

\section{INTRODUCTION}

Microbial infection of the root canal system is the key etiology behind endodontic infections. The main objective of pediatric endodontic therapy is to maintain the primary teeth in the dental arch and to facilitate the proper eruption of permanent successors. The role of a clinician is to maintain strict asepsis in the vital pulp situation and to attain antisepsis for nonvital and necrosed pulps. Hence, the microbial reduction or elimination from root canals of infected teeth is vital for successful treatment. ${ }^{1}$ Endodontic infection in primary teeth is polymicrobial in nature, comprising of aerobic, anaerobic, and facultative microbes. ${ }^{2}$

The endodontic instrumentation alone is inefficient in eliminating the microflora from the root canals of primary teeth mechanically owing to their structural complexity. The dependence on irrigating solutions becomes more crucial, especially in primary teeth due to thin dentinal walls, complex morphology, and tortuous root canals. ${ }^{3}$

An ideal endodontic irrigant should exhibit powerful antimicrobial activity, dissolve organic tissue remnants, disinfect the root canal space, flush out debris from the instrumented root canals, provide lubrication, and have no cytotoxic effects on the periradicular tissues. ${ }^{1}$ Numerous formulations have been used in root canals of permanent teeth with their respective advantages and disadvantages. However, there are lack of studies related to efficacy of root canal irrigants used in primary teeth. $5.25 \%$ sodium hypochlorite and chlorhexidine are the commonly used irrigants. Each has its own merits and demerits. Sodium hypochlorite shows significant antimicrobial activity and tissue-dissolving property. However, it is toxic to periapical tissues, allergic, and has unpleasant taste. ${ }^{4}$ Chlorhexidine shows substantivity and lower cytotoxicity. But it lacks the ability to dissolve the necrotic pulp tissue and
${ }^{1}$ Department of Pedodontics and Preventive Dentistry, Institute of Dental Studies and Technologies, Modinagar, Ghaziabad, Uttar Pradesh, India

${ }^{2-6}$ Department of Pedodontics, College of Dental Sciences, Davangere, Karnataka, India

Corresponding Author: Shagun Sinha, Department of Pedodontics and Preventive Dentistry, Institute of Dental Studies and Technologies, Modinagar, Ghaziabad, Uttar Pradesh, India, Phone: +91 9743493032, e-mail: shagun3.sinha@gmail.com

How to cite this article: Sinha S, Korishettar R, Parameswarappa P, et al. Comparative Evaluation of Antimicrobial Activity of Quercetin against Endodontic Pathogens in Primary Molars. CODS J Dent 2018;10(1): 7-10.

Source of support: Nil

Conflict of interest: None

exhibits limited activity against gram-negative bacteria. ${ }^{5}$ Hence, the search for an ideal irrigating solution for endodontic treatment in primary and permanent teeth is still ongoing.

In the past few years, researchers have shown great interest in the investigation of natural products to determine their efficacy for various medical purposes, because of several advantages like ample material sources, ease of use, better efficacy, and less side effects. ${ }^{6}$ Various endodontic irrigants derived from natural sources have been tested for their efficacy in the recent years. Some of these include propolis, neem, aloe vera, garlic, triphala, and grape seed extract. ${ }^{7}$

Flavonoids are natural pigments with variable phenolic structures and are found in fruit, vegetables, grains, bark, roots, 
stems, flowers, tea, and wine. The four main groups of flavonoids include flavones, flavanones, catechins, and anthocyanins. The flavones are characterized by a planar structure because of a double bond in the central aromatic ring. ${ }^{8}$ One of the widely researched flavonoid, quercetin, is a member of this group. It is found in abundance in onions, apples, broccoli, and berries. The key effect of flavonoids is their ability to scavenge oxygen-derived free radicals. Various studies have reported that flavonoids possess anti-inflammatory, antiallergic, antiviral, and anticarcinogenic properties. Quercetin is known to exhibit significant antimicrobial property. ${ }^{9}$ Its antibacterial action probably depends on disruption of the membrane and inactivation of extracellular proteins by forming irreversible complexes, but the exact mechanism remains unclear. ${ }^{6}$

However, there is paucity of literature on evaluation of the antimicrobial efficacy of quercetin against endodontic pathogens. Thus, the present study was conducted to comparatively evaluate the antimicrobial efficacy of $1 \%(\mathrm{w} / \mathrm{v})$ and $2 \%(\mathrm{w} / \mathrm{v})$ quercetin against endodontic pathogens in primary teeth.

\section{Materials and Methods}

\section{Preparation of Irrigant Solution}

$1 \%(\mathrm{w} / \mathrm{v})$ and $2 \%(\mathrm{w} / \mathrm{v})$ solution of quercetin was prepared by adding $1 \mathrm{~g}$ and $2 \mathrm{~g}$ of pure quercetin powder (Purebulk, USA) to $100 \mathrm{~mL}$ of $50 \%$ dimethylsulfoxide (DMSO) (Himedia, Mumbai) solution, respectively. $0.2 \%$ chlorhexidine solution (Indoco Remedies, India) was used as the control irrigant.

\section{Source of Data}

A total of 15 patients with age ranging from 4 years to 7 years, reporting to the Department of Pedodontics and Preventive Dentistry with nonvital carious primary molars, were selected for the study. Ethical clearance was taken from the institutional review board. A written consent was obtained from the parents prior to the procedure. Study design was experimental, in vivo study. Children with nonvital carious primary molar were included in the study. Molars showing less than two-thirds of root length, mobility, or resorption and children with systemic illness were excluded from the study.

\section{Collection of Samples}

Samples of endodontic microflora were isolated from root canals of primary molars. The sample thus obtained simulated the flora at the periapex of the tooth.

The oral cavity and the tooth of interest were disinfected with povidone iodine to obtain a sterile field. Coronal access was gained to the root canals of the involved teeth under rubber dam isolation. Access was gained and all carious tissues were removed using a large round bur. In mandibular primary molar distal canal and in maxillary primary molar palatal canal were chosen for the sampling procedure. The pulp was extirpated with the help of a no. 15 sterile barbed broach. Following this, a no. 15 sterile paper point was inserted into the canal, $1 \mathrm{~mm}$ short of radiographic apex and held there for 2 minutes to obtain a sample of the mixed culture of microorganisms. This paper point was then transferred to a sterile test tube containing $2 \mathrm{~mL}$ of brain heart infusion (BHI) broth. The samples were taken to the microbiology lab for further processing. $^{10}$

To check the antimicrobial efficacy of the irrigants, the Agar disk diffusion method was used. The irrigants were divided into three groups: group I: $0.2 \%$ chlorhexidine solution, group II: $1 \%(\mathrm{w} / \mathrm{v})$ solution of quercetin, and group III: $2 \%(\mathrm{w} / \mathrm{v})$ solution of quercetin.

\section{Culture of Samples}

Blood agar Petri plates were prepared and $200 \mu \mathrm{L}$ of microbial inoculum was spread uniformly onto the agar plates using a sterile cotton swab. Following this, $30 \mu \mathrm{L}$ of irrigant solution from each of the three groups was poured onto three corresponding sterile filter paper disks ( $6 \mathrm{~mm}$ diameter) using a pipette. The disks were then placed equidistantly on the agar Petri plates using a sterile tweezer. For each sample, one Petri dish was cultured under the microaerophilic condition in a candle jar at $37^{\circ} \mathrm{C}$ for 24 hours. After 24 hours, the diameter of maximum growth inhibition around each disk was measured and recorded using a divider and a vernier caliper (Fig. 1). ${ }^{11}$

\section{Statistical Analysis}

The mean inhibition zone for all three groups was determined in millimeter. The statistical analysis of data was done using Tukey post hoc tests.

\section{Results}

Table 1 and Figure 2 show the mean zone of inhibition for all the three groups under the microaerophilic condition. The highest inhibition zone was shown by group I, that is, $13.13 \pm 0.74$, followed by group III, with $12.00 \pm 0.65$, and the lowest zone was observed in group II, i.e., $8.53 \pm 0.63$.

Table 2 shows comparison between the mean zone of inhibition $(\mathrm{mm} \pm \mathrm{SD})$ for all the three groups under the microaerophilic condition. When group I was compared with group II and group III, group II was compared with group I and group III, and group III was compared with group I and group II, statistically significant difference was noted $(p=0.000)$.

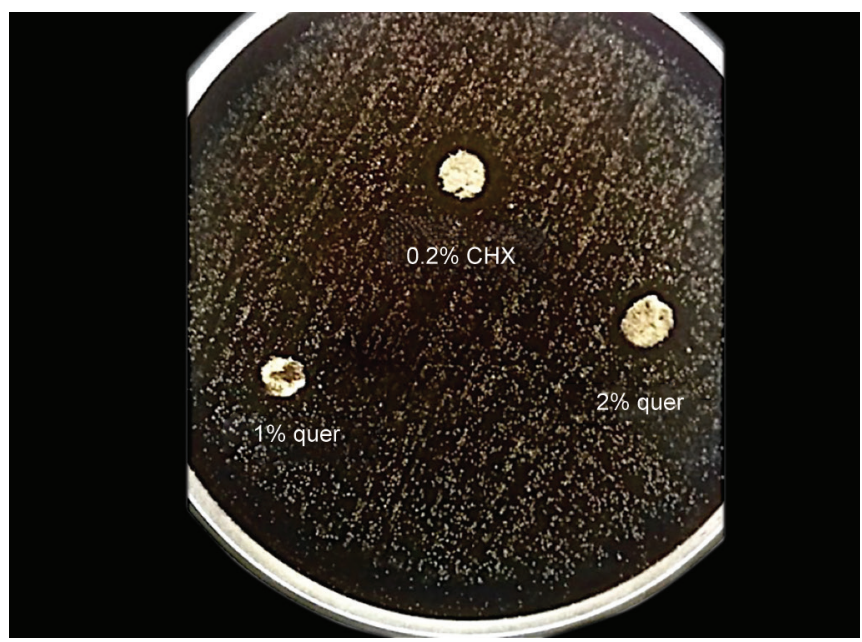

Fig. 1: Cultured Petri plate showing zone of inhibition under microaerophilic conditions

Table 1: Mean zone of inhibition $(\mathrm{mm})$ under microaerophilic conditions

\begin{tabular}{llll}
\hline Group & $n$ & Mean & Std. deviation \\
\hline Group I & 15 & 13.1333 & 0.74322 \\
Group II & 15 & 8.5333 & 0.63994 \\
Group III & 15 & 12.0000 & 0.65465 \\
Total & 45 & 11.2222 & 2.08772 \\
\hline
\end{tabular}

$p<0.05$, significant 


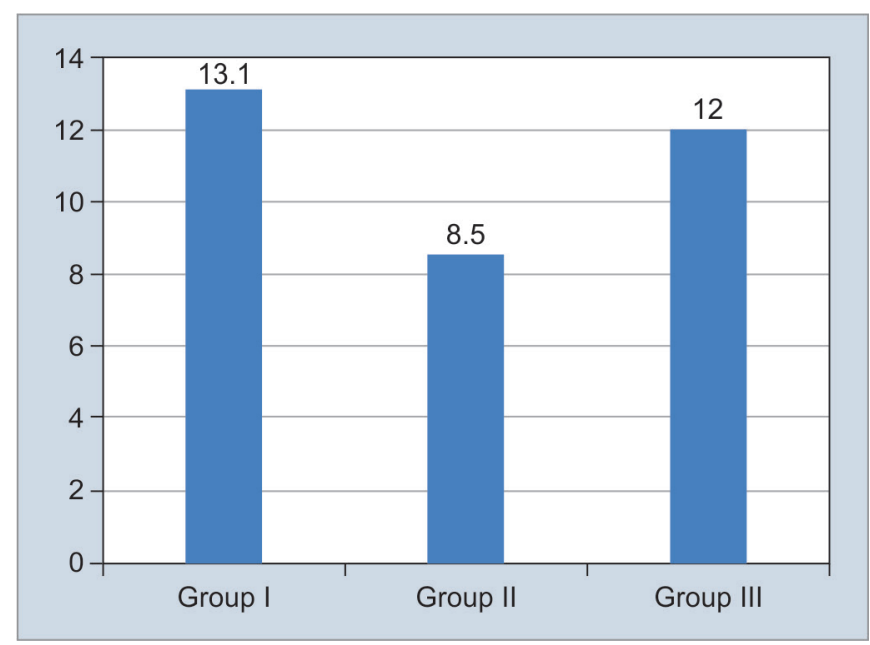

Fig. 2: Mean zone of inhibition $(\mathrm{mm})$ under microaerophilic conditions

Table 2: Multiple comparison between groups using Tukey's post hoc tests under microaerophilic conditions

\begin{tabular}{llcll}
\hline (I) & $(J)$ & $\begin{array}{l}\text { Mean difference } \\
(I-J)\end{array}$ & Std. error & Sig. \\
\hline Group I & Group II & 4.60000 & 0.24859 & 0.000 \\
& Group III & 1.13333 & 0.24859 & 0.000 \\
Group II & Group I & -4.60000 & 0.24859 & 0.000 \\
& Group III & -3.46667 & 0.24859 & 0.000 \\
Group III & Group I & -1.13333 & 0.24859 & 0.000 \\
& Group II & 3.46667 & 0.24859 & 0.000 \\
\hline
\end{tabular}

$p<0.05$, significant

\section{Discussion}

Microbial reduction is the key objective of the endodontic therapy, which consecutively promotes the normal healing process of the periodontal tissues. ${ }^{1}$ During the biomechanical preparation of teeth with periapical lesions, endodontic irrigants play an important role in disinfecting the root canals.

Primary root canal infections are polymicrobial, typically dominated by anaerobic bacteria. ${ }^{2}$ The most frequently isolated microorganisms before root canal treatment include gramnegative anaerobic rods, gram-positive anaerobic cocci, grampositive anaerobic and facultative rods, Lactobacillus species, and gram-positive Streptococcus species. ${ }^{12}$ Ruviére et al. evaluated the microbial species present in root canals of infected primary teeth. The microbes associated with root canals of teeth showing irreversible pulpitis were Campylobacter rectus (87\%), Gemella morbillorum (78\%), Streptococcus gordonii (71\%), Capnocytophaga ochracea (69\%), Treponema denticola (58\%), and Streptococcus intermedius (49\%). Whereas, the most prevalent species in root canals with teeth showing apical periodontitis were $C$. rectus (90\%), T. denticola (88\%), S. intermedius (77\%), G. morbillorum (73\%), Streptococcus oralis (67\%), C. ochracea (63\%), S. gordonii (55\%), Streptococcus mitis (51\%), and Leptotrichia buccalis (51\%). ${ }^{13}$ A high percentage of failure after endodontic treatment of teeth with periapical lesions has been related to implications of the microbial origin. ${ }^{14}$ The obligate anaerobes are rather easily eradicated during root canal treatment. On the other hand, facultative bacteria such as Enterococci and Staphylococcus, once established, are more likely to survive chemomechanical instrumentation of the root canal. ${ }^{1}$
Many natural plants and their extracts have been used for medical purpose for centuries and their biological and pharmacological effects have attracted more and more investigations in recent years. ${ }^{6}$ Flavonoids, the main bioactive compound found in many natural products, such as green tea and cranberry, are known to be effective antimicrobial agents against a wide array of microorganisms. Quercetin is a natural flavonoid that exists in various parts of plants. It is known to exhibit antimicrobial properties. It inhibits gram-positive and gramnegative bacteria through inactivating extracellular proteins. Apart from antimicrobial activity, it also displays other beneficial effects: antitumor, antioxidant, anti-inflammatory, and antiviral. ${ }^{9}$

The present study aimed at comparative evaluation of the antimicrobial activity of $0.2 \%$ chlorhexidine, $1 \%(\mathrm{w} / \mathrm{v})$ quercetin, and $2 \%(\mathrm{w} / \mathrm{v})$ quercetin against endodontic pathogens isolated from root canals of primary molars. As $0.2 \%$ chlorhexidine is a commonly used irrigant in endodontics with significant antimicrobial activity, it was used as positive control. ${ }^{15,16}$ Since quercetin is known to exhibit antimicrobial properties, but till date no study has been done to evaluate its efficacy against endodontic pathogens in primary teeth, it was chosen for the present study. As quercetin is insoluble in water, 50\% DMSO was used as a solvent. Dimethylsulfoxide has no effect on microorganisms and it also potentiates the action of the extract, as stated by Bhargava et al. ${ }^{11}$ The samples were obtained from nonvital primary molars using a paper point. The palatal canal for maxillary molar and the distal canal for mandibular molar were chosen for collecting the sample as they are wider and easily accessible. ${ }^{10}$ Antimicrobial efficacy was evaluated using the disk diffusion test and the maximum inhibition zone was measured after 24 hours. ${ }^{11}$

Results of the present study showed that under the microaerophilic condition, $0.2 \%$ chlorhexidine $(13.1 \mathrm{~mm})$ exhibited maximum antimicrobial activity, followed by $2 \%$ quercetin $(12 \mathrm{~mm})$, and least antimicrobial activity was shown by $1 \%$ quercetin $(8.5 \mathrm{~mm})$. On comparison between groups, statistically significant difference was present.

In our study, $0.2 \%$ chlorhexidine showed the highest antimicrobial activity against endodontic pathogens. This finding is consistent with studies done by Bulacio Mde et al. who stated that $0.2 \%$ chlorhexidine showed significant antimicrobial activity against $E$. fecalis with an inhibition zone of $14 \mathrm{~mm} .{ }^{15} \mathrm{~A}$ study done by Ferraz et al. also showed that $0.2 \%$ chlorhexidine exhibited significant antibacterial effect against different endodontic pathogens, with the inhibition zone ranging from $4 \mathrm{~mm}$ to $12 \mathrm{~mm} .{ }^{16}$ The antimicrobial effect of chlorhexidine is due to the interaction of the positive charge of the chlorhexidine molecule and the negatively charged phosphate groups on microbial cell walls, thereby altering the cell's osmotic equilibrium. This increases the permeability of the cell wall, which allows the $\mathrm{CHX}$ molecule to penetrate into the bacteria. ${ }^{17}$

Also, in the present study, $2 \%$ quercetin showed significant antimicrobial activity, close to that of $0.2 \%$ chlorhexidine against endodontic pathogens under microaerophilic conditions. This is in agreement with the study done by Shu et al. who concluded that quercetin exhibits significant antimicrobial activity against several oral infectious pathogens including S. mutans, S. sobrinus, L. acidophilus, S. sanguis, A. actinomycetemcomitans, and P. intermedia. ${ }^{9}$

$\mathrm{Li}$ and $\mathrm{Xu}$ have also stated that quercetin shows potent antimicrobial efficacy against periodontal pathogens including A. actinomycetemcomitans, A. viscosus, P. gingivalis, F. nucleatum, and 
A. naes/undii. ${ }^{18}$ Patra et al. concluded in their study that quercetin possesses antibacterial activity against S. mutans. ${ }^{19}$ A study was by Contini et al. showed that quercetin shows antibacterial property against S. mutans and Staphylococcus aureus. ${ }^{20}$ According to Cowan, the antibacterial mechanism of quercetin probably depends on disruption of the membrane and inactivation of extracellular proteins by forming irreversible complexes but the exact mechanism remains unclear. ${ }^{6}$

\section{CONCLUSION}

In the present study, significant antimicrobial activity against endodontic pathogens in primary molars was shown by $0.2 \%$ chlorhexidine and $2 \%(\mathrm{w} / \mathrm{v})$ quercetin, whereas $1 \%(\mathrm{w} / \mathrm{v})$ quercetin showed low antimicrobial efficacy. On comparison between groups, $0.2 \%$ chlorhexidine showed maximum antimicrobial efficacy, followed by $2 \%(\mathrm{w} / \mathrm{v})$ quercetin, and lastly by $1 \%(\mathrm{w} / \mathrm{v})$ quercetin. In terms of antimicrobial efficacy against endodontic pathogens in primary molars, quercetin shows promising results. However, since our study is first of its kind, more studies are required before coming to a definite conclusion.

\section{References}

1. Zehnder M. Root canal irrigants. J Endod 2006;32(5):389-398. DOI: 10.1016/j.joen.2005.09.014.

2. Rana V, Baba SM, Pandey A. Bacteriology of infected deciduous root canal - a review. A PJSR 2009;2(2):45-48.

3. Thomas AM, Chandra S, Chandra $S$, et al. Elimination of infection in pulectomized deciduous teeth: a short-term study using iodoform paste. J Endod 1994;20(5):233-235. DOI: 10.1016/S00992399(06)80284-0.

4. Clarkson RM, Moule AJ. Sodium hypochlorite and its use as an endodontic irrigant. Aust Dent J 1998;43(4):250-256. DOI: 10.1111/ j.1834-7819.1998.tb00173.x. Review.

5. Khademi A, Usefian E, Feizianfard M. Tissue dissolving ability of several endodontic irrigants on bovine pulp tissue. Iran Endod J 2007;2(2):65-68.

6. Majorie MC. Plant products as antimicrobial agents. Clin Microbiol Rev 1990;12(4):564-582.

7. Vinothkumar TS, Rubin MI, Balaji L, et al. In vitro evaluation of five different herbal extracts as an antimicrobial endodontic irrigant using real-time quantitative polymerase chain reaction. J Conserv Dent 2013;16(2):167-170. DOI: 10.4103/0972-0707.108208.
8. Middleton EJ. Effect of plant flavonoids on immune and inflammatory cell function. Adv Exp Med Biol 1998;439:175-182. DOI: 10.1007/9781-4615-5335-9_13.

9. Shu Y, Liu Y, Li L, et al. Antibacterial activity of quercetin on oral infectious pathogens. Afr J Microbiol Res 2011;5(30):5358-5361.

10. Verma MK, Pandey RK, Khanna R, et al. The antimicrobial effectiveness of $25 \%$ propolis extract in root canal irrigation of primary teeth. J Indian Soc Pedod Prev Dent 2014;32(2):120-124. DOI: 10.4103/09704388.130786.

11. Bhargava K, Kumar T, Aggarwal S, et al. Comparative evaluation of the antimicrobial efficacy of neem, green tea, triphala and sodium hypochlorite: an in vitro study. J Dent Res Rev 2015;2(1):13-16. DOI: 10.4103/2348-2915.154638.

12. Shen Y, Stojicic S, Qian W, et al. The synergistic antimicrobial effect by mechanical agitation and two chlorhexidine preparations on biofilm bacteria. J Endod 2010;36(1):100-104. DOI: 10.1016/j.joen.2009. 09.018.

13. Ruviére DB, Leonardo MR, da Silva LA, et al. Assessment of the microbiota in root canals of human primary teeth by checkerboard DNA-DNA hybridization. J Dent Child 2007;74(2):118-123.

14. Sundqvist G, Figdor D, Persson S, et al. Microbiologic analysis of teeth with failed endodontic treatment and the outcome conservative treatment. Oral Surg Oral Med Oral Pathol 1998;85(1):86-93. DOI: 10.1016/S1079-2104(98)90404-8.

15. Bulacio Mde L, Cangemi R, Cecilia M, et al. In vitro antibacterial effect of different irrigating solutions on Enterococcus faecalis. Acta Odontol Latinoam 2006;19(2):75-80.

16. Ferraz CC, Gomes BP, Zaia AA, et al. Comparative study of the antimicrobial efficacy of chlorhexidine gel, chlorhexidine solution and sodium hypochlorite as endodontic irrigants. Braz Dent J 2007;18(4):294-298. DOI: 10.1590/S0103-64402007000400004.

17. Gomes BPFA, Souza SFC, Ferraz CCR, et al. Effectiveness of $2 \%$ chlorhexidine gel and calcium hydroxide against Enterococcus faecalis in bovine root dentine in vitro. Int Endod J 2003;36(4):267-275. DOI: 10.1046/j.1365-2591.2003.00634.x.

18. Li M, Xu Z. Quercetin in a lotus leaves extract may be responsible for antibacterial activity. Arch Pharm Res 2008;31(5):640-644. DOI: 10.1007/s12272-001-1206-5.

19. Patra JK, Kim ES, Oh K, et al. Antibacterial effect of crude extract and metabolites of Phytolacca americana on pathogens responsible for periodontal inflammatory diseases and dental caries. BMC Complement Altern Med 2014;14:343. DOI: 10.1186/1472-6882-14-343.

20. Helena T-CSilvia, José SM, Evandro W, et al. Antimicrobial activity of flavonoids and steroids isolated from two Chromolaena species. Rev Bras Cienc Farm 2003;39(4):403-408. DOI: 10.1590/S151693322003000400007. 